Pacific Journal of Mathematics

THE SATURATION OF $k$-ANALYTIC RINGS AND
TOPOLOGICAL EQUIVALENCE OF ASSOCIATED ANALYTIC
SET GERMS

ULRICH DeEP 


\title{
THE SATURATION OF $k$-ANALYTIC RINGS AND TOPOLOGICAL EQUIVALENCE OF ASSOCIATED ANALYTIC SET GERMS
}

\section{ULRICH DAEPP}

\begin{abstract}
The objective of this paper is to adapt the theory of saturation as developed by Oscar Zariski to the case of $k$-analytic rings. For the most part $k$ is an algebraically closed field of positive characteristic. We think that saturation can be helpful in the definition of equisingularity. The results beneath show that some necessary conditions for such a task are fulfilled in this particular case. We do however not go so far as to actually define equisingularity.
\end{abstract}

In $\S 1$ we give definitions and some results concerning $k$-analytic rings and their associated analytic set germs. In $\S 2$ we apply the concept of saturation as defined in [18]. In particular, we show that under certain conditions-which can be met in our frameworkthe saturation of a $k$-analytic ring is defined and is again $k$-analytic. In $\S 3$ we show the topological relation between the analytic set germs associated with a $k$-analytic ring and its saturation, respectively. This generalizes the results of a paper by A. Seidenberg [17]. There the same theory is developed in the case $k=\boldsymbol{C}$. In the last section we get some partial results of showing that the multiplicity of a $k$-analytic ring and its saturation are the same. However, some restrictive conditions have to be put on the ring.

Concepts and notations not defined explicitly follow those used by Zariski and Samuel [20] and [21].

This paper contains part of my thesis. I wish to thank Professor W. E. Kuan for suggesting the topic and for his continued encouragement.

I wish to thank the referee for his or her helpful suggestions which lead in particular to a better proof of Lemma 2.4.

1. Preliminaries. If $k$ is a field with a nontrivial complete valuation, then $k\left[\left[X_{1}, \cdots, X_{n}\right]\right]$ denotes the ring of formal power series over $k$ in $n$ variables, $k\left[\left\{X_{1} \cdots, X_{n}\right\}\right]$ denotes the subring consisting of all convergent power series, see [1] p. 7. By an analytic ring over $k$ we mean a $k$-algebra which is the $k$-homomorphic image of some convergent power series ring with coefficients in $k$. A local ring $A$ is called $k$-analytic if it contains a subring $B$ such that $B$ is an analytic ring over $k$ and $A$ is a finite $B$-module. If $k$ is alge- 
braically closed-as will mostly be the case in this paper--then a ring $A$ is $k$-analytic if and only if it is an analytic ring over $k$, Corollary 1.5, p. 30 of [2].

We will need the following, later on:

LEMMA 1.1. Let $k$ be an algebraically closed, complete valued field. Let $A$ be an integral domain containing $k\left[\left\{x_{1}, \cdots, x_{d}\right\}\right]$ as subring where the $x_{i}$ 's are analytically independent. If $A$ is a finite module over $k\left[\left\{x_{1}, \cdots x_{d}\right\}\right]$, then $A$ is an analytic ring over $k$.

Proof. In view of the above remark it is enough to show that $A$ is local. By Hensel's lemma as stated in 12.2, p. 95 of [1], $k\left[\left\{x_{1}, \cdots, x_{d}\right\}\right]$ is Henselian. Theorem 43.12, p. 183 of [12] gives now the required conclusion.

We will need the normalization theorem for convergent power series rings in the following form. (Compare e.g., Theorem 45.5, on p. 193 of [12].)

LEMMA 1.2. Let $k$ be an algebraically closed, complete and nontrivially valued field and $A$ a local ring of dimension $d$.

If $A$ is an analytic ring over $k$ and $x_{1}, \cdots, x_{d}$ is any system of parameters of $A$ then $k\left[\left\{x_{1}, \cdots, x_{d}\right\}\right] \subseteq A, k\left[\left\{x_{1}, \cdots, x_{d}\right\}\right]$ is $k$-isomorphic to a convergent power series ring in $d$ variables and $A$ is a finite $k\left[\left\{x_{1}, \cdots, x_{d}\right\}\right]-$ module.

Conversely, if $k\left[\left\{x_{1}, \cdots, x_{d}\right\}\right] \subseteq A, A$ is a finite $k\left[\left\{x_{1}, \cdots, x_{d}\right\}\right]-$ module and $\operatorname{dim}\left(k\left[\left\{x_{1}, \cdots, x_{d}\right\}\right]\right)=d$, then $A$ is an analytic ring over $k$ and $x_{1}, \cdots, x_{d}$ is a system of parameters of $A$.

Whenever we have a complete and nontrivially valued field $k$ which is algebraically closed and a ring $A$ which is $k$-analytic then Lemma 1.2 allows us to write $A=k\left[\left\{x_{1}, \cdots, x_{d}\right\}\right]\left[y_{1}, \cdots, y_{s}\right]$. Here $x_{1}, \cdots, x_{d}$ is any system of parameters of $A$.

We denote by $\bar{V}$ an analytic set germ at the origin of $k^{n}$. Two germs $\bar{V}_{1}$ and $\bar{V}_{2}$ are topologically equivalent if there are representatives $\left(V_{1}, U_{1}\right)$ and $\left(V_{2}, U_{2}\right)$ and continuous maps $\varphi: V_{1} \cdots, V_{2}$ and ir: $V_{2} \rightarrow V_{1}$ such that $\psi \varphi$ and $\varphi_{\psi}$ s are the identity maps on $V_{1}$ and $V_{2}$ respectively. If in addition $\varphi$ and $\psi^{\prime}$ extend to the open sets $U_{1}$ and $U_{2}$ respectively and are analytic on them then $\bar{V}_{1}$ and $\bar{V}_{2}$ are said to be analytically equivalent. For more details compare [7], where these concepts are developed in the case $k=C$.

If $A$ is a $k$-analytic ring with representation $A=k\left[\left\{X_{1}, \cdots, X_{n}\right\}\right] / 2$ I wherc $F_{1}, \cdots, F_{s}$ generate $\mathfrak{A}$ then we can associate an analytic set 
germ of $k^{n}$ with $A$ as follows: Let $U$ be a neighborhood of $O$ in $k^{n}$ on which $F_{1}, \cdots, F_{s}$ converge. Let $W$ be the set of common zeros of $F_{1}, \cdots, F_{s}$ in $U$. The analytic set germ associated with $A$ is the class containing the representative $(W, U)$. We denote it by $\bar{V}_{A}$. It does not depend on the particular set of generators we took. Also, the radical of $\mathfrak{A}$ gives rise to the same set germ. The analytic set germ does not depend on the particular representation of $A$ :

LEMMA 1.3. Let $k$ be an algebraically closed, complete and nontrivially valued field. If $k\left[\left\{X_{1}, \cdots, X_{n}\right\}\right] / \mathscr{R}$ and $k\left[\left\{Y_{1}, \cdots, Y_{m}\right\}\right] / \mathfrak{L}^{2}$ are $k$-isomorphic then their associated analytic set germs are analytically equivalent.

The proof is left to the reader.

The following well known result and its corollary give some information about the dimension of the ambient space of an analytic set germ associated with a given analytic ring over $k$.

Lemma 1.4. Let $k$ be a complete, nontrivially valued and algebraically closed field. Let $A$ be an analytic ring over $k$ and $y_{1}, \cdots, y_{s}$ a set of generator's for the maximal ideal in $A$. Then $A=k\left[\left\{y_{1}, \cdots, y_{s}\right\}\right]$.

COROLLARY 1.5. Let $k$ be a complete, nontrivially valued and algebraically closed field. Let $A$ be an analytic ring over $k$ of embedding dimension $n$. We have associated analytic set germs in $k^{l}$ for all $l \geqq n$.

2. Strongly separating systems of parameters. In this section we will show that a saturation in the sense of Zariski [18] pp.961693 of a $k$-analytic ring can be constructed. However, certain conditions which will be specified later have to be satisfied. Also, we will have to choose a suitable field $K$ with respect to which to define the saturation. The saturated ring is then again $k$-analytic.

We will need the following lemma:

LeMma 2.1. Let $k$ be a valued field which is perfect and let $A$ be a reduced analytic ring over $k$. The integral closure $\bar{A}$ of $A$ in its total ring of quotients $\Phi(A)$ is a finite A-module.

$\Phi(A)$ is the direct sum of fields $\Phi(A)=F_{1} \oplus \cdots \oplus F_{s}$ and if $\varepsilon_{i}$ is the identity of $F_{i}$ as an element in $\Phi(A)$, then $\bar{A}$ is the direct sum of the integral closures of the $A \varepsilon_{i}$ 's in the $F_{i}$ 's. 
Proof. Since $A$ is a finite module over a convergent power series ring we have by [12], 45.6 on p. 194 that $A$ is a Weierstrass ring and hence is in particular pseudo-geometric. That is, if $p \in$ $\operatorname{Spec}(A)$ then the integral closure of $A / p$ in its field of quotients is a finite $A / p$-module. The lemma follows now from 19.23 , p. 167 of [1].

The total ring of quotients of a Noetherian ring, and hence of a ring $A$ which is reduced and analytic over $k$ can be described more precisely. Namely $\Phi(A)=\Phi\left(A / p_{1}\right) \oplus \cdots \oplus \Phi\left(A / p_{s}\right)$ where $p_{1}, \cdots, p_{s}$ denote the minimal primes of $A$.

We introduce the following definition:

Definition 2.2. Suppose $A=k\left[\left\{x_{1}, \cdots, x_{d}\right\}\right]\left[y_{1}, \cdots, y_{m}\right]$ where $x_{1}, \cdots, x_{d}$ is a system of parameters for $A$ and the $y_{1}, \cdots, y_{m}$ are integral over $k\left[\left\{x_{1}, \cdots, x_{d}\right\}\right]$. The system of parameters $x_{1}, \cdots, x_{d}$ is said to be strongly separating if there exist $m$ monic polynomials $P_{i}(Z)$ in $k\left[\left\{x_{1}, \cdots, x_{d}\right\}\right][Z]$ such that $P_{i}\left(y_{i}\right)=0$ for $1 \leqq i \leqq m$ and which are separable considered as polynomials over the field $k\left(\left\{x_{1}, \cdots, x_{d}\right\}\right)$.

An analytic ring over $k$ which has a strongly separating system of parameters is called strongly separable.

LEMMA 2.3. Let $k$ be an algebraically closed, complete and nontrivially valued field. Let $A$ be a reduced and equidimensional $k$-analytic ring and $\Phi(A)=F_{1} \oplus \cdots \oplus F_{s}$ its total ring of quotients. $\varepsilon_{i}$ denotes the unit of $F_{i}$ in $\Phi(A)$. If $x_{1}, \cdots, x_{d}$ is a strongly separating system of parameters of $A$ then $F_{i}$ is a finite algebraic and separable extension of $\varepsilon_{i} k\left(\left\{x_{1}, \cdots, x_{d}\right\}\right)$ for $1 \leqq i \leqq s$.

Proof. We first consider the case where $A$ is a domain. We have then the following commutative diagram where all maps are the obvious inclusions.

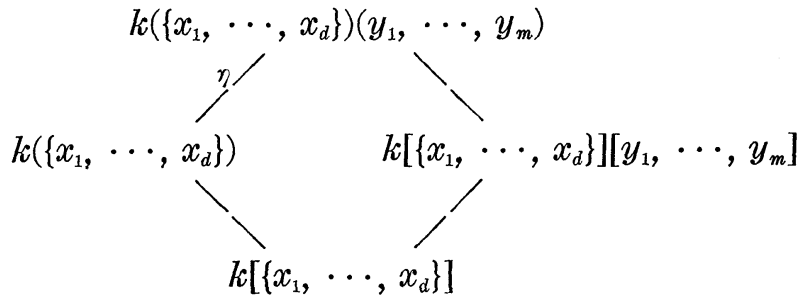

Clearly $k\left(\left\{x_{1}, \cdots, x_{d}\right\}\right)\left(y_{1}, \cdots, y_{m}\right)=\Phi\left(k\left[\left\{x_{1}, \cdots, x_{d}\right\}\right]\left[y_{1}, \cdots, y_{m}\right]\right)=\Phi(A)$. The monic irreducible polynomial of $y_{i}$ over the field $k\left(\left\{x_{1}, \cdots, x_{d}\right\}\right)$ 
divides $P_{i}$ which is separable. Hence, $\eta$ is separable.

We can now look at the general case and denote the minimal primes of $A$ by $p_{1}, \cdots, p_{s}$. We claim that $k\left[\left\{x_{1}, \cdots, x_{d}\right\}\right] \cap p_{i}=(0)$ for $1 \leqq i \leqq s$. To see this we notice that for each $p_{i}$ we have a chain of prime ideals $p_{i} \subset q_{1} \subset \cdots \subset q_{d}$ of length $d+1$ in $A$. Contracting this chain to $k\left[\left\{x_{1}, \cdots, x_{d}\right\}\right]$ we get another proper chain in this ring of length $d+1$, hence $p_{i} \cap k\left[\left\{x_{1}, \cdots, x_{d}\right\}\right]=(0)$. This establishes the claim.

Consider the following diagram:

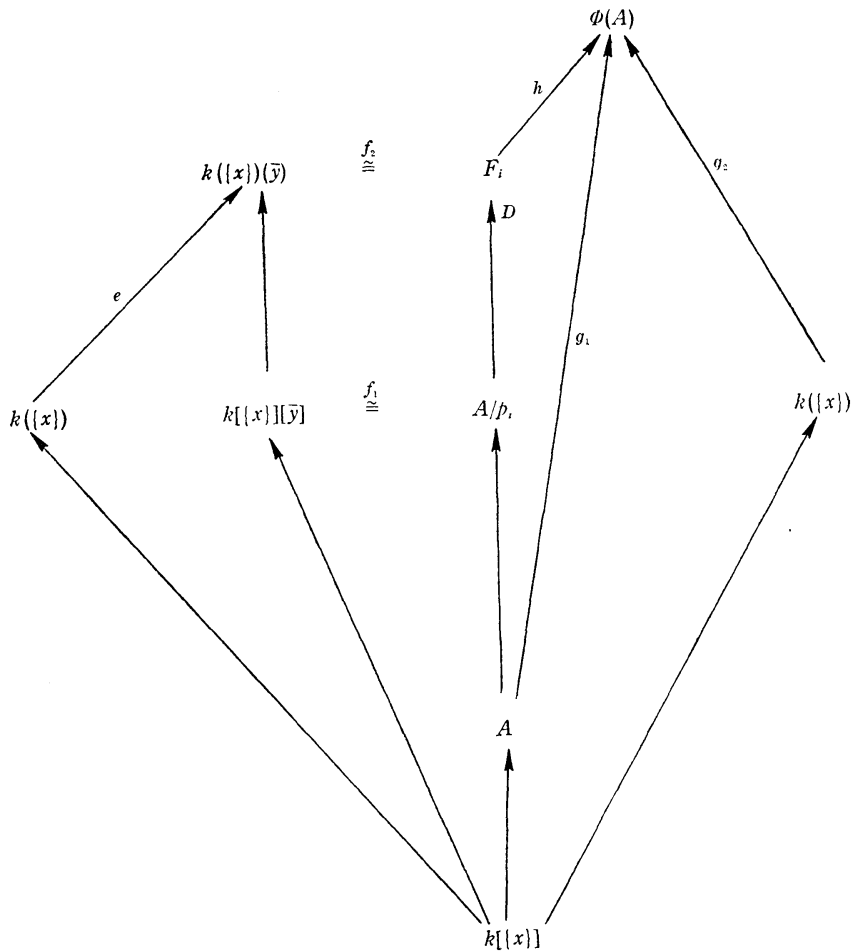

$x$ stands for $x_{1}, \cdots, x_{d}$ and $y$ for $y_{1}, \cdots, y_{m}$. $\bar{y}$ denotes $y+p_{i}$.

We define the maps in the diagram above as follows:

$$
f_{1}\left(\sum a_{i_{1} \cdots i_{m}} y_{1}^{i_{1}} \cdots y_{m}^{i_{m}}+p_{i}\right)=\sum a_{i_{1} \cdots i_{m}} \bar{y}_{1}^{i_{1}} \ldots \bar{y}_{m}^{i_{m}} .
$$

Since $k\left[\left\{x_{1}, \cdots, x_{d}\right\}\right] \cap p_{i}=(0)$ this is an isomorphism. $f_{2}$ is defined analogously. $g_{1}(a)=\left(a+p_{1}, \cdots, a+p_{s}\right) . g_{2}$ is defined in the same way. $e$ and $h$ are the natural embeddings. It is clear that all subdiagrams commute, except for diagram $D$.

If $P_{i}(Z)$ is an integral relation for $y_{i}$ over $k\left[\left\{x_{1}, \cdots, x_{d}\right\}\right]$ then it is also one for $\bar{y}_{i}$ over the same ring. The special case treated first shows now that $F_{i}$ is a finite separable algebraic extension of $k\left(\left\{x_{1}, \cdots, x_{d}\right\}\right)$. The lemma will be proven if we show that 
$h f_{2} e(k(\{x\}))=\varepsilon_{i} g_{2}(k(\{x\}))$. But $h f_{2} e(a)=h\left(a+p_{i}\right)=\left(0, \cdots, a+p_{i}, \cdots\right.$, $0)=\varepsilon_{i}\left(a+p_{1}, \cdots, a+p_{s}\right)=\varepsilon_{i} g_{2}(a)$.

Our next goal is to show that all $k$-analytic rings are strongly separable.

LEMma 2.4. Let $k$ be an algebraically closed, complete and nontrivially valued field. Let $A=k\left[\left\{x_{1}, \cdots, x_{n}\right\}\right]$ be a reduced and equidimensional k-analytic ring of dimension $d . p_{1}, \cdots, p_{s}$ denote its minimal primes. There is a k-linear transformation

$$
y_{i}=\sum_{i=1}^{n} a_{i j} x_{j}, 1 \leqq i \leqq d, a_{i j} \in k
$$

such that $y_{1}, \cdots, y_{d}$ is a system of parameters of $A$ and the quotientfields $\Phi\left(A / p_{i}\right)$ are finite algebraic and separable over $\varepsilon_{i} k\left(\left\{y_{1}, \cdots, y_{d}\right\}\right)$ for all $i, 1 \leqq i \leqq s$.

Proof. This lemma is a generalization of 24.5 in [1], p. 201. The proof given there can be changed to our situation. Therefore, we will only sketch the proof and indicate the changes. See [1] for more details.

For each minimal prime $p_{l}$ we set $\mathscr{L}_{l}=\Phi\left(A / p_{l}\right)$. We can then construct $0 \neq P_{l}(B)=P_{l}\left(\cdots, B_{i j}, \cdots\right) \in \mathscr{L}_{l}^{P}[B]$. Here $p=\operatorname{char}(k)$ and $B=\cdots, B_{i j}, \cdots, 1 \leqq i \leqq d, 1 \leqq j \leqq n$ are indeterminants. For each $l, 1 \leqq l \leqq s$ one can find $0 \neq Q_{l}(B) \in k[B]$ such that $Q_{l}\left(a_{i j}\right) \neq 0$ implies $P_{l}\left(a_{i j}\right) \neq 0$ where $a_{i j} \in k$. Set $Q(B)=Q_{1}(B) Q_{2}(B) \cdots Q_{s}(B)$. Then $0 \neq Q(B) \in k[B]$. According to 23.5 of [1] there is a $k$-linear transformation $y_{i}=\sum_{j=1}^{n} a_{i j} x_{j}, 1 \leqq i \leqq d$, such that $y_{1}, \cdots, y_{d}$ is a system of parameters for all $A / p_{i}$ and hence for $A$, and also $Q\left(a_{i j}\right) \neq 0$. Hence $P_{l}\left(a_{i j}\right) \neq 0$ for all $l$. It can be shown that $\mathscr{L}_{l}=\mathscr{L}_{l}^{P}\left(k\left(\left\{y_{1}, \cdots\right.\right.\right.$, $\left.\left.\left.y_{d}\right\}\right)\right)$. Hence, by Theorem 8 of [20] p. 69, we get that $\mathscr{L}_{l}$ is separable over $k\left(\left\{y_{1}, \cdots, y_{d}\right\}\right)$ for all $l, 1 \leqq l \leqq s$.

THEOREM 2.5. Let $k$ be an algebraically closed and nontrivially valued field. A reduced and equidimensional k-analytic ring is strongly separable.

Proof. Write $A=k\left[\left\{y_{1}, \cdots, y_{d}\right\}\right]\left[x_{1}, \cdots, x_{m}\right]$ where $y_{1}, \cdots, y_{d}$ is a system of parameters as constructed in Lemma 2.4. For $p \in \operatorname{Ass}_{A}(0)$ we have $\operatorname{Irr}_{A / p}\left(\bar{x}_{1}, \varepsilon k\left(\left\{y_{1}, \cdots, y_{d}\right\}\right)\right)=Z^{n}+\bar{a}_{n-1} Z^{n-1}+\cdots$ the irreducible polynomial of $\bar{x}_{1}$ of $A / p$ over $\varepsilon k\left(\left\{y_{1}, \cdots, y_{d}\right\}\right)$. Let $a_{i}$ in $k\left(\left\{y_{1}, \cdots, y_{d}\right\}\right)$ be the unique preimage of $\bar{a}_{i}$ and set $P_{p}(Z)=Z^{n}+$ $a_{n-1} Z^{n-1}+\cdots$. Let $t$ be the number of minimal primes containing $x_{1}$. Set 


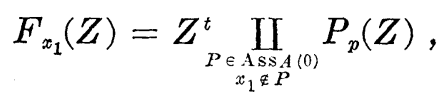

$F_{x_{2}}(Z), \cdots, F_{x_{m}}(Z)$ are constructed analogously. One checks that those are the required separable polynomials for $x_{1}, \cdots, x_{m}$.

The central Lemma 2.4 can also be obtained using some theorems of Scheja and Storch, [16], instead. On the other hand, our Lemmas 2.3 and 2.4 show that their seemingly weaker definition of separability and our strong separability are equivalent for the considered type of rings. Therefore, their result 4.2 in [16] follows from our Theorem 2.5.

The following example serves two purposes. First, it shows that not every system of parameters is strongly separating. Second, it makes apparent that separating with respect to a prime, as used in [16], and strongly separating are different requirements for a system of parameters.

EXAMPLE 2.6. Let $k$ be an algebraically closed, complete and nontrivially valued field of characteristic $p>2 . \quad X_{1}^{2}-X_{2}$ and $X_{1}^{p}-X_{2}$ are two prime elements of $R=k\left[\left\{X_{1}, X_{2}\right\}\right]$. Set $P=$ $\left(X_{1}^{2}-X_{2}\right) R, Q=\left(X_{1}^{p}-X_{2}\right) R$ and $I=P \cap Q$. The example we want to consider is $A=k\left[\left\{X_{1}, X_{2}\right\}\right] / I$. Setting $p=P / I$ and $q=Q / I$ we have $\operatorname{Ass}_{A}(0)=\{p, q\}$. Clearly $A$ is a reduced $k$-analytic ring, equidimensional of dimension 1 . We denote by $x_{1}$ and $x_{2}$ the images of $X_{1}$ and $X_{2}$ respectively under the natural projection. An easy check shows that $x_{1}$ is a strongly separating parameter. $x_{2}$ on the other hand projects to a separating parameter in $A / p$ but to a nonseparating one in $A / q$.

We come now to the main result of this section. We will use the notation of saturation as in [18], p. 963 .

THEOREM 2.7. Let $k$ be an algebraically closed, complete and nontrivially valued field. Let $A$ be an equidimensional and reduced k-analytic ring. Then there exists a system of parameters $x_{1}, \cdots, x_{d}$ of $A$ such that the saturation of $A$ with respect to $k\left(\left\{x_{1}, \cdots, x_{d}\right\}\right)$ is defined. We will denote it by $\widetilde{A}_{\left(x_{1}, \cdots, x_{d}\right)}$. For each system of parameters for which the saturation exists it is again a $k$-analytic ring of dimension $d$.

Proof. We first have to check conditions (a) through (e) as stated in [18] p.962: (a) is part of the assumptions; (b) follows from Lemma 2.1; for (c): here $K=k\left(\left\{x_{1}, \cdots, x_{d}\right\}\right)$ and obviously $1 \in k$. 
According to Lemma 2.3 it is enough to choose a strongly separating system of parameters in order to satisfy (d). That we can find such a system follows from Theorem 2.6. For (e): $k\left[\left\{x_{1}, \cdots, x_{d}\right\}\right] \subseteq$ $A \cap k\left(\left\{x_{1}, \cdots, x_{d}\right\}\right)=R$ and that $A$ is integral over $R$ follows from Lemma 1.2.

Now suppose that $x_{1}, \cdots, x_{d}$ is a system of parameters for which the saturation is defined. We denote it by $\widetilde{A}_{x}$ and $\bar{A}$ stands for the integral closure of $A$ in $\Phi(A)$. From Lemma 2.1 and the fact that $A$ is Noetherian we conclude that $\widetilde{A}_{x}$ is a finite $A$-module. From Lemma 1.2 it follows that $\widetilde{A}_{x}$ is finite over $k\left[\left\{x_{1}, \cdots, x_{d}\right\}\right]$. Since $k\left[\left\{x_{1}, \cdots, x_{d}\right\}\right]$ is integrally closed in its quotient field we have $R=A \cap k\left(\left\{x_{1}, \cdots, x_{d}\right\}\right)$ and it follows from [18], 4.1, p. 997 that $\operatorname{Spec}\left(\widetilde{A}_{x}\right) \rightarrow \operatorname{Spec}(A)$ is radicial. Hence $\widetilde{A}_{x}$ is a local ring and $\operatorname{dim}\left(\widetilde{A}_{x}\right)=\operatorname{dim}(A)=d$. By the second part of Lemma 1.2 we get that $\widetilde{A}_{x}$ is a $k$-analytic ring.

Corollary 2.8. Let $k$ and $A$ be as in Theorem 2.7. If $x_{1}, \cdots, x_{d}$ is a system of parameters for which the saturation exists then it is strongly separating.

Proof. The proof of Theorem 2.5 shows that Zariski's condition (d) implies that the system of parameters is strongly separating.

3. Topological equivalence. As we just have shown the saturation of a $k$-analytic ring is again $k$-analytic, provided one takes a strongly separating system of parameters. As explained in $\S 1$, one can associate analytic set germs with both rings. The purpose of this section is to show that these germs are topologically equivalent.

Suppose we have two analytic rings over $k, A$ and $A^{\prime}, A \subseteq A^{\prime}$ and $A^{\prime}$ is finite over $A$, say $A^{\prime}=A\left[y_{1}, \cdots, y_{m}\right]$. If $A=k\left[\left\{x_{1}, \cdots, x_{n}\right\}\right]$ then there is an associated analytic set germ $\bar{V}_{A}$ in $k^{n} . A^{\prime}=$ $k\left[\left\{x_{1}, \cdots, x_{n}, y_{1}, \cdots, y_{m}\right\}\right]$ gives then rise to a set germ $\bar{V}_{A^{\prime}}$ in $k^{n+m}$. In this situation we say that $\bar{V}_{A^{\prime}}$ lies over $\bar{V}_{A}$.

If $D \in A$ then we will write $D\left(x_{1}, \cdots, x_{n}\right)$ for a representation of $D$ in $k\left[\left\{x_{1}, \cdots, x_{n}\right\}\right] . \quad D\left(X_{1}, \cdots, X_{n}\right)$ is then the power series which has the same coefficients as $D\left(x_{1}, \cdots, x_{n}\right)$ but has the ring elements $x_{i}$ replaced by the indeterminates $X_{i}$. If $a=\left(a_{1}, \cdots, a_{n}\right) \in k^{n}$ then $D(a)$ simply means $D\left(X_{1}, \cdots, X_{n}\right)$ evaluated at $X_{i}=a_{i}$.

Lemma 3.1. Let $k$ be an algebraically closed, complete and nontrivially valued field of characteristic $p>0$. Let $A \subseteq A^{\prime}$ be two $k$-analytic rings such that $A^{\prime}$ is a finite A-module. Further assume 
that there are $D \in A, D \neq 0$ and $\alpha \in N$ such that $D a^{p^{\alpha}} \in A$ for all $a \in A^{\prime}$. Then the analytic set germs $\bar{V}_{A}$ and $\bar{V}_{A^{\prime}}$, where $\bar{V}_{A^{\prime}}$ lies over $\bar{V}_{A}$, have representatives $\left(V_{A}, U\right)$ and $\left(V_{A^{\prime}}, U^{\prime}\right)$ such that above every $a \in V_{A}$ with $D(a) \neq 0$ there lies one and only one point of $V_{A^{\prime}}$.

Proof. We first prove the uniqueness: If $A^{\prime}=A\left[y_{1}, \cdots, y_{m}\right]$ then we have $D y_{i}^{p^{\alpha}}=g_{i} \in A$ for $1 \leqq i \leqq m$. We take a set of defining functions for $V_{A^{\prime}}$ and include among them the $m$ functions $D(X) Y_{i}^{p^{\alpha}}-g_{i}(X) . D(X)$ and $g_{i}(X)$ are defined as explained previous to the statement of the theorem. Let $a=\left(a_{1}, \cdots, a_{n}\right) \in V_{A}$ with $D(a) \neq 0$. Let $b$ and $c$ be two different points above $a$ in $V_{A^{\prime}}, b=$ $\left(a_{1}, \cdots, a_{n}, b_{1}, \cdots, b_{m}\right)$ and $c=\left(a_{1}, \cdots, a_{n}, c_{1}, \cdots, c_{m}\right)$. Since $b$ and $c$ are in $V_{A^{\prime}}$ we have $D(a) b_{i}^{p^{\alpha}}-g_{i}(\alpha)=0$ or $b_{i}^{p^{\alpha}}-g_{i}(a) / D(a)=0$ for $1 \leqq i \leqq m$. In the same way $c_{i}^{p^{\alpha}}-g_{i}(a) / D(a)=0$ for $1 \leqq i \leqq m$. Hence $b_{i}$ and $c_{i}$ are both solutions of the equation $Z^{p^{\alpha}}-r=0$. Since $k$ is algebraically closed there is $s \in k$ such that $s^{p^{\alpha}}=r$ and therefore $Z^{p^{\alpha}}-r=(Z-s)^{p^{\alpha}}=0$. Hence the equation has only one solution and we conclude that $b_{i}=c_{i}$ for $1 \leqq i \leqq m$, which shows that $b=c$.

It remains to show the existence. If $A^{\prime}=k\left[\left\{X_{1}, \cdots, X_{n}, Y_{1}, \cdots\right.\right.$, $\left.\left.Y_{m}\right\}\right] / \mathfrak{B}$ let $F_{1}(X, Y), \cdots, F_{s}(X, Y)$ be a set of generators for $\mathfrak{B}$. Since $A^{\prime}$ is a finite $A$-module we may assume that $F_{i}(X, Y) \in$ $k[\{X\}][Y]$. Take $\rho$ big enough such that

$$
\begin{gathered}
{[D(X)]^{\rho}\left[F_{i}\left(X,\left[g_{1}(X) / D(X)\right]^{p^{-\alpha}}, \cdots,\left[g_{m}(X) / D(X)\right]^{p^{-\alpha}}\right)\right]^{p^{\alpha}}} \\
=G_{i}(X) \in k[\{X\}] \text { for } 1 \leqq i \leqq s .
\end{gathered}
$$

We let $U_{\varepsilon}^{\prime}=\left\{\left(b_{1}, \cdots, b_{n+m}\right) \in k^{n+m}|| b_{i} \mid<\varepsilon\right\}$. We choose $\varepsilon$ small enough such that all $F_{i}(X, Y), 1 \leqq i \leqq s$ converge on $U_{\varepsilon}^{\prime}$ and consider $\left(V_{A^{\prime}}, U_{\varepsilon}^{\prime}\right)$. Now let $\omega>0$ such that $\omega \leqq \varepsilon,\left[g_{i}(a) / D(a)\right]^{p^{-\alpha}}<\varepsilon$ for all $i, 1 \leqq i \leqq m$ whenever $a \in U_{\omega}=\left\{\left(a_{1}, \cdots, a_{n}\right) \in k^{n}|| a_{i} \mid<\omega\right\}$ and such that all $G_{i}(X), 1 \leqq i \leqq s$ and $D(X)$ are convergent on $U_{\omega}$. We can now include the $G_{i}(X)$ among the generators for an analytic set $\left(V_{A}, U_{\omega}\right)$. If $a \in V_{A}$ and $D(a) \neq 0$ then we let $b=\left(a,\left[g_{1}(a) / D(a)\right]^{p^{-\alpha}}, \cdots\right.$, $\left.\left[g_{m}(a) / D(a)\right]^{p^{-\alpha}}\right) . \quad b \in U_{\varepsilon}$ and $\left[F_{i}(b)\right]^{p^{\alpha}}=G_{i}(a) / D(a)^{\rho}=0$ for $1 \leqq i \leqq s$. Hence $F_{i}(b)=0$ which implies that $b \in\left(V_{A^{\prime}}, U_{\varepsilon}\right)$.

We now state two lemmas which are well known in the complex case. We will point out at the end of this section why the usual short proofs will not work in this case (see Theorem 3.6).

LEMMA 3.2. Let $k$ be an algebraically closed and valued field. Let $1 \leqq m \leqq n$ and $\alpha_{1}, \cdots, \alpha_{n} \in k$ ordered such that $\left|\alpha_{1}\right| \leqq \cdots \leqq\left|\alpha_{n}\right|$. Then there is a nonnegative real valued function $\varphi_{m}$ on $\left(\boldsymbol{R}^{+}\right)^{m}$ such 
that the following two conditions are satisfied:

(1) $\left|\alpha_{m}\right| \leqq \varphi_{m}\left(t_{1}, \cdots, t_{m}\right)$ where $t_{i}=\left|s_{n-i+1}\left(\alpha_{1}, \cdots, \alpha_{n}\right)\right|$ and $s_{j}$ denotes the $j$ th elementary symmetric polynomial.

(2) $\varphi_{m}\left(x_{1}, \cdots, x_{k}\right) \rightarrow 0$ as $x_{1} \rightarrow 0, \cdots, x_{k} \rightarrow 0$.

A proof can be found in [10], pp. 102-104. Kneser proved the lemma there only for $k=\boldsymbol{C}$. But the reader checks easily that the proof works for any algebraically closed and valued field.

COROLLARY 3.3. Let $k$ be an algebraically closed and valued field, $f_{1}(x)=x^{n}+a_{n-1} x^{n-1}+\cdots+a_{0}$ and $f_{2}(x)=x^{n}+b_{n-1} x^{n-1}+\cdots+b_{0}$, $a_{i}, b_{i} \in k$. Denote the roots of $f_{2}$ by $x_{1}, \cdots, x_{n}$ and suppose $c$ is $a$ root of $f_{1}$ of multiplicity $m$. Then for every $\varepsilon>0$ there is a $\delta>0$ such that if $\left|a_{i}-b_{i}\right|<\delta, 0 \leqq i \leqq n-1$, then $\left|x_{1}-c\right|<\varepsilon, \cdots$, $\left|x_{m}-c\right|<\varepsilon$ after appropriate enumeration of the roots of $f_{2}$.

This follows from the lemma above. A detailed proof is in [4], pp. $33 / 34$.

We are now equipped to prove the following theorem.

TheOREM 3.4. Let $A=k\left[\left\{x_{1}, \cdots, x_{n}\right\}\right]$ and $A^{\prime}=k\left[\left\{x_{1}, \cdots, x_{n}\right.\right.$, $\left.y_{1}, \cdots, y_{m}\right\}$ ] where $k$ is an algebraically closed complete and nontrivially valued field. Suppose that

(1) $A^{\prime}$ is a finite $A$-module and $A \subseteq A^{\prime}$.

(2) $A^{\prime}$ is reduced.

(3) $A^{\prime}$ is a radicial extension of $A$.

Let $\bar{V}_{A}$ and $\bar{V}_{A^{\prime}}$ be associated analytic set germs in $k^{n}$ and $k^{n+m}$ respectively. Then there are representatives $\left(V_{A}, U\right)$ and $\left(V_{A^{\prime}}, U^{\prime}\right)$ such that the projection $\pi: k^{n+m} \rightarrow k^{n}$ induces a homeomorphism on the analytic sets.

Proof. If $\operatorname{char}(k)=0$ then $k=C$ and the theorem is identical to Theorem 9 of [17], p. 429. Hence we assume throughout the proof that $\operatorname{char}(k)>0$.

Let $P_{1}, \cdots, P_{\mathrm{s}}$ be the minimal primes of $A^{\prime}$. Since $A^{\prime}$ is reduced $P_{1} \cap \cdots \cap P_{s}=(0)$ is an irredundant primary decomposition. Let $p_{i}=P_{i} \cap A$, then $(0)=p_{1} \cap \cdots \cap p_{s}$. Suppose we could leave out one of the primes, say $p_{1}$. Then $p_{1} \supset p_{2} \cap \cdots \cap p_{s}$ and we have $p_{1} \supseteqq p_{i}$ for some $i, 2 \leqq i \leqq s$, say $p_{1} \supseteqq p_{2}$. By the going up theorem, [3], 5.11, p. 62, there is a prime $Q$ in $A^{\prime}$ such that $P_{2} \subseteq Q$ and $Q \cap A=p_{1}$. Since the extension is radicial we have $Q=P_{1}$ and hence $P_{2} \subseteq P_{1}$ which is a contradiction. This shows that $p_{1}, \cdots, p_{s}$ are exactly the minimal primes of $A$ and $A$ is therefore also reduced. We have 
now $\Phi(A)=\Phi\left(A / p_{1}\right) \oplus \cdots \oplus \Phi\left(A / p_{s}\right)$ and $\Phi\left(A^{\prime}\right)=\Phi\left(A^{\prime} / P_{1}\right) \oplus \cdots \oplus \Phi\left(A^{\prime} / P_{s}\right)$. The map $\left(a+p_{i}\right) /\left(b+p_{i}\right) \rightarrow\left(a+P_{i}\right) /\left(b+P_{i}\right)$ determines a natural embedding of $\Phi(A)$ in $\Phi\left(A^{\prime}\right)$.

Next we show that there are analytic sets $\left(V_{A}, U\right)$ and $\left(V_{A^{\prime}}, U^{\prime}\right)$ such that above every point of $V_{A}$ there lies exactly one point of $V_{A^{\prime}}$. Hence the projection is a bijection between $\left(V_{A}, U\right)$ and $\left(V_{A^{\prime}} \cap\left(U \times k^{n}\right), U^{\prime} \cap\left(U \times k^{n}\right)\right)$. We will show this by induction on the dimension $d$ of $A$. Suppose $d=0$, then $\left(V_{A}, U\right)$ and $\left(V_{A^{\prime}}, U^{\prime}\right)$ both contain only the origin and the statement follows trivially. Let us now assume that $d>0$ and that the existence of two sets lying above each other in the required way is established for all smaller dimensions. Since $A \subseteq A^{\prime} \subseteq \Phi\left(A^{\prime}\right)$ we can write $A^{\prime}=\sum_{1}^{t} A a_{i}$ where $a_{i} \in \Phi\left(A^{\prime}\right)$. Since the extension is radicial, each $\Phi\left(A^{\prime} / P_{i}\right)$ is purely inseparable over $\Phi\left(A / p_{i}\right)$ and hence there is an $\alpha \in N$ such that $a_{i}^{p^{\alpha}}=r_{i} / s_{i} \in \Phi(A), r_{i}, s_{i} \in A$, for all $i, 1 \leqq i \leqq t$. Let $D=\prod_{1}^{t} s_{i} \in A$. Then $D \neq 0$ and $D a^{p^{\alpha}} \in A$ for all $a \in A^{\prime}$. By Lemma 3.1 we can find $\left(V_{A}, U\right)$ and $\left(V_{A^{\prime}}, U^{\prime}\right)$ such that there is exactly one element in $V_{A^{\prime}}$ above each element $a \in V_{A}$ if $D(a) \neq 0$. We consider the analytic subset of $V_{A}$ on which $D$ vanishes. Let $I^{\prime}=\operatorname{rad}\left(D \cdot A^{\prime}\right), I=I^{\prime} \cap A$, $\bar{A}=A / I$ and $\bar{A}^{\prime}=A^{\prime} / I^{\prime}$. Clearly, (1), $\bar{A}^{\prime}$ is a finite $\bar{A}$-module and, (2), $\bar{A}^{\prime}$ is reduced. But also (3) holds, the extension $\bar{A} \leqq \bar{A}^{\prime}$ is radicial. For suppose $p \in \operatorname{Spec}(\bar{A})$, then it corresponds to some $\widetilde{p} \in$ $\operatorname{Spec}(A)$ with $I \subseteq \widetilde{p}$. If $P$ and $Q$ are primes in $\bar{A}^{\prime}, P \cap \bar{A}=Q \cap \bar{A}=p$ then consider the corresponding primes $\widetilde{P}$ and $\widetilde{Q}$ in $A^{\prime}$. Now $\widetilde{P} \cap$ $A=\widetilde{Q} \cap A=\widetilde{p}$. This contradicts the radiciality of $A^{\prime}$ over $A$, hence there is at most one prime above $p$ in $\bar{A}^{\prime}$. Further we have $\bar{A} / p \cong$ $A / \widetilde{p}$ and $A^{\prime} / p \cong A^{\prime} / \widetilde{P}$. Since $A / \widetilde{p} \rightarrow A^{\prime} / \widetilde{P}$ is purely inseparable so is $\bar{A} / p \rightarrow \bar{A}^{\prime} / P$. In conclusion we have that $\operatorname{Spec}\left(\bar{A}^{\prime}\right) \rightarrow \operatorname{Spec}(\bar{A})$ is radicial. Since $D$ is not a zero divisor in $A^{\prime}$ we have $\operatorname{dim} \bar{A}^{\prime}<$ $\operatorname{dim} A^{\prime}$. By the induction hypothesis we have two sets $\left(V_{\bar{A}}, W\right)$ and $\left(V_{\bar{A}^{\prime}}, W^{\prime}\right)$ which lie above each other in the required way and therefore do the sets $\left(V_{A}, U \cap W\right)$ and $\left(V_{A^{\prime}}, U^{\prime} \cap W^{\prime}\right)$.

It remains to show that the projection restricted to the analytic sets and its inverse are topological maps. For the projection this is clear and for $\left(x_{1}, \cdots, x_{n}\right) \rightarrow\left(x_{1}, \cdots, x_{n}, y_{1}, \cdots, y_{m}\right)$ this follows fairly easily from Corollary 3.3. For details see [4], pp. 37/38.

The main theorem of this section follows now easily.

THEOREM 3.5. Let $k$ be an algebraically closed, complete and nontrivially valued field. Let $A$ be an equidimensional and reduced $k$-analytic ring. Let $x_{1}, \cdots, x_{d}$ be a strongly separating system of parameters of $A$ and $\widetilde{A}_{x}$ the saturation with respect to this system. Then the two associated analytic set germs are topologically equi- 
valent.

In fact, the homeomorphism can be induced by the natural projection of the ambient spaces if the representations of the rings are chosen so that the associated set germs lie above each other.

Proof. By Theorem 2.7, $\widetilde{A}_{x}$ is k-analytic and hence a finite $k[\{x\}]$-module. This shows that $\widetilde{A}_{x}$ is finite over $A$. Since $\widetilde{A}_{x} \subseteq \Phi(A)$ and $A$ is reduced, we conclude that $\widetilde{A}_{x}$ is reduced. The fact that $\widetilde{A}_{x}$ is radicial is proven in Theorem 4.1, [18], p.997. We can now apply Theorem 3.4 and get the second half of our theorem. Lemma 1.3 shows that the particular representation of the ring does not matter and therefore finishes up the proof.

Lemma 3.2 and the part in the proof to Theorem 3.4 which establishes the continuity of the map can be proven much more easily in the case when $k=C$. The shorter proofs are based on the fact that every bounded sequence in $C$ has a convergent subsequence. The following theorem shows that we do not have this fact available in our situation and that we can therefore not hope to adapt the usual proofs.

Recall that a space is called sequentially compact if and only if every sequence has a convergent subsequence.

THEOREM 3.6. Let $k$ be an algebraically closed, nontrivially valued field of positive characteristic and let $A_{\alpha}=\{x \in k|| x \mid \leqq \alpha\}$, where $\alpha \in \boldsymbol{R}^{+}$. Then $A_{\alpha}$ is not sequentially compact.

Proof. Since $A_{\alpha}$ is metric it is paracompact; see [5], p. 186, Theorem 5.3. By [9], p. 162, E), part (d), $A_{\alpha}$ is sequentially compact if and only if it is countably compact. The latter is the case if and only if $A_{\alpha}$ is compact, [5], p. 230, Corollary 3.4.

Now suppose $A_{\alpha}$ is sequentially compact and hence compact. Then $k$ is locally compact, since addition is continuous. Since $\operatorname{char}(k)>0$ the valuation is nonarchimedean and from Theorem 1 of [14], p. 245, it follows that the valuation is discrete, that is $|k-\{0\}|$ is a cyclic subgroup of the positive real numbers. Say $|x|$ is a generator of this group. We can assume that $|x|>1$. It is easy to see that $|x|=\min \{|y|>1 \mid y \in k\}$. Since $k$ is algebraically closed there is $a \in k$ such that $a^{2}=x$ and therefore $1<|a|<|x|$. This contradiction shows that $A_{\alpha}$ cannot be sequentially compact.

4. Multiplicities. Another necessary condition for equisingularity, an algebraic one, is that the local rings have the same multiplicity. This requirement is discussed in this section. 
Recall that $\mathfrak{A}$ and $\mathscr{B}$ are two ideals and $\mathfrak{A} \subseteq \mathscr{B}$ then $\mathfrak{A}$ is said to be a reduction of $\mathscr{B}$ provided we can find a positive integer $r$ such that $\mathscr{B} \mathfrak{A}^{r}=\mathfrak{Q}^{r+1}$.

THEOREM 4.1. Suppose $k$ is an algebraically closed, complete and nontrivially valued field. Let $A$ be an equidimensional and reduced k-analytic ring. Suppose $x_{1}, \cdots, x_{d}$ is a strongly separating system of parameters and the ideal it generates is a reduction of the maximal ideal of $A$. Denote by $F_{j}^{*}$ the least Galois extension of $k\left(\left\{x_{1}, \cdots, x_{d}\right\}\right)$ which contains $\Phi\left(A / p_{j}\right)$, where $\left\{p_{1}, \cdots, p_{s}\right\}=\operatorname{Ass}_{A}(0)$. Further assume that char $(k)$ and $\left[F_{j}^{*}: k\left(\left\{x_{1}, \cdots, x_{d}\right\}\right)\right]$ are relatively prime for all $j, 1 \leqq j \leqq s$. Then $A$ and $\widetilde{A}_{x}$ have the same multiplicity.

One can adapt the proof of Theorem 4.1 of [19], pp. 455-460. In our case one has to use Theorem 1 of [13] to show the validity for nondomains directly. The possibility for that is already remarked on p. 460 of [19]. Some other small changes are necessary but they do not justify the reproduction of this long proof here. For a completely written out proof in our case, see [4], pp. 42-48.

Recall that the system of parameters $x_{1}, \cdots, x_{d}$ of a local ring $(A, m)$ is said to be transversal if $e\left(\left(x_{1}, \cdots, x_{d}\right) A\right)=e(m)$.

CoRollary 4.2. Let $k$ be as in Theorem 4.1. Let $A$ be a k-analytic integral domain. Let $x_{1}, \cdots, x_{d}$ be a (strongly) separating and transversal system of parameters. Denote by $F^{*}$ the least Galois extension of $k\left(\left\{x_{1}, \cdots, x_{d}\right\}\right)$ which contains $\Phi(A)$. Suppose that $\operatorname{char}(k)$ and $\left[F^{*}: k\left(\left\{x_{1}, \cdots, x_{d}\right\}\right)\right]$ are relatively prime. Then $e(A)=e\left(\widetilde{A}_{x}\right)$.

Proof. The statement follows from Theorem 4.1 if we can show that $\left(x_{1}, \cdots, x_{d}\right) A$ is a reduction of the maximal ideal in $A$. This follows from [15], p. 16, Theorem 3.2, if we can show that all minimal primes in the completion of $A$ are of dimension $d=\operatorname{dim}(A)$. Theorem 44.1 of [12], p. 188 shows that $A$ is analytically irreducible, that is, the completion is even a domain.

It is not known to me if anything can be said about the multiplicity if the parameters do not generate a reduction of the maximal ideal. The same question was raised in [19], p. 460 for the characteristic zero algebroid case. However, there are nontrivial cases to which the above theorem applies. To show this, is the purpose of the following example. 
EXAMPLE 4.3. This is a ring satisfying all conditions of Corollary 4.2 and having nontrivial saturation. Let $k$ be an algebraically closed, nontrivially valued field with $\operatorname{char}(k)>3$. We consider $A=$ $k[\{X, Y\}] /\left(Y^{3}+Y^{2}+X^{2}+2 X Y\right)$. It is easy to check that $A$ is a domain and $\operatorname{dim}(A)=1$. We write $x$ and $y$ for $X$ and $Y$ modulo the relation. $m=(x, y)$ is the maximal ideal and $x$ is a system of parameters since $m^{2} \subseteq(x)$. Hence $A=k[\{x\}][y]$ where $y^{3}+y^{2}+2 x y+$ $x^{2}=0 . \quad(x)$ is a reduction of $m$ since $m^{2}(x)=m^{3}$. One also checks that it is (strongly) separating. $f(Z)=Z^{3}+Z^{2}+2 x Z+x^{2}$ is irreducible in $k[\{x\}][Z]$ and hence in $k(\{x\})[Z]$. Therefore $[\Phi(A): k(\{x\})]=$ $[k(\{x\})(y): k(\{x\})]=\operatorname{deg}(f(Z))=3 . \quad F^{*}$ is the splitting field of $f(Z)$ over $k(\{x\})$, hence $\operatorname{char}(k)$ and $\left[F^{*}: k(\{x\})\right]$ are relatively prime. That $A$ is not saturated can be seen as follows: If it were saturated then $A$ were an Arf ring since $\operatorname{dim}(A)=1$ and $A$ is Cohen-Macaulay, [11], p. 682, Corollary 5.3. Then we would have $\operatorname{dim}_{A / m}\left(m / m^{2}\right)=$ $e(A)$, by [11], p. 661, Theorem 2.2. Since $\left(Y^{3}+Y^{2}+2 X Y+X^{2}\right) \subseteq$ $(X, Y)^{2}$ and $k[\{X, Y\}]$ is a regular local ring of dimension 2, we have $\operatorname{dim}_{A / m}\left(m / m^{2}\right)=2$. To calculate the multiplicity of $A$ we use [21], p. 299, Corollary 1 and get $e(x A)=3$. Since $(x)$ is a reduction of $m=(x, y)$ we have $e(A)=3$. This contradiction shows that $A$ is not saturated.

\section{REFERENCES}

1. S. S. Abhyankar, Local Analytic Geometry, Academic Press, New York and London, 1964.

2. S. S. Abhyankar and M. van der Put, Homomorphisms of analytic rings, J. reine angew. Math., 242 (1970), 26-60.

3. M. F. Atiyah and I. G. MacDonald, Introduction to Commutative Algebra, AddisonWesley, Reading, Mass., 1969.

4. U. Daepp, Saturations of an analytic ring over an algebraically closed field, Thesis, Michigan State University, 1979.

5. J. Dugundji, Topology, Allyn and Bacon, Boston, 1966.

6. A. V. Geramita and C. Small, Introduction to Homological Methods in Commutative Rings, Queen's Papers, Kingston, Ontario, 1976.

7. R. C. Gunning, Lectures on Complex Analytic Varieties: The Local Parametrization Theorem, Princeton University Press, Princeton, N. J., 1970.

8. N. Jacobson, Basic Algebra I, Freeman, San Francisco, 1974.

9. J. L. Kelley, General Topology, D. van Nostrand, New York, 1955.

10. H. Kneser, Zur Stetigkeit der Wurzeln einer algebraischen Gleichung, Math. Z.,

48 (1942), 101-104.

11. J. Lipman, Stable ideals and Arf rings, Amer. J. Math., 93 (1971), 649-685.

12. M. Nagata, Local Rings, Krieger, Huntington, N. Y., reprint 1975.

13. M. Nagata, Note on a paper of Samuel concerning asymptotic properties of ideals, Memoirs of the College of Science, University of Kyoto, Ser. A, Vol. XXX, Math., No. 2, (1957), 165-175.

14. E. U. Obienu, On local compactness in nonarchimedean normed linear spaces, Math. Japonica, 23, No. 2 (1978), 245-247.

15. D. Rees, $\alpha$-transforms of local rings and a theorem on multiplicities of ideals, 
Proc. Cambridge Phil. Soc., 57 (1961), 8-17.

16. G. Scheja and U. Storch, Differentielle Eigenschaften der Lokalisierungen analytischer Algebren, Math. Ann., 197 (1972), 137-170.

17. A. Seidenberg, Saturation of an analytic ring, Amer. J. Math., 94 (1972), 424-430. 18. O. Zariski, Studies in equisingularities III, Saturation of local rings and equisingularity, Amer. J. Math., 90 (1968), 961-1023.

19. O. Zariski. General theory of saturation and of saturated local rings III, Saturation in arbitrary dimension and, in particular, saturation of algebroid hypersurfaces, Amer. J. Math., 97 (1975), 415-502.

20. O. Zariski and P. Samuel, Commutative Algebra, Volume 1, Springer-Verlag, New York, Heidelberg and Berlin, 1975.

21. Commutative Algebra, Volume 2, van Nostrand, Princeton, N. J., 1960.

Received December 23, 1979.

Michigan State University

East Lansing, MI 48824 



\section{PACIFIC JOURNAL OF MATHEMATICS}

\section{EDITORS}

DONALD BABBITT (Managing Editor)

University of California

Los Angeles, CA 90024

Hugo RossI

University of Utah

Salt Lake City, UT 84112

C. C. Moore and Arthur Agus

University of California

Berkeley, CA 94720
J. DugundJI

Department of Mathematics

University of Southern California

Los Angeles, CA 90007

R. FinN and J. MiLgRAM

Stanford University

Stanford, CA 94305

\section{ASSOCIATE EDITORS}
R. ARENS
E. F. BECKENBACH
B. H. NeumanN
F. WOLF
K. YoSHIDA

\section{SUPPORTING INSTITUTIONS}

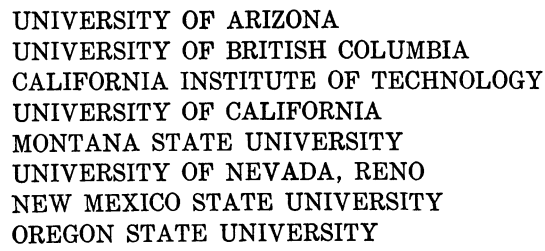

UNIVERSITY OF ARIZONA

UNIVERSITY OF BRITISH COLUMBIA CALIFORNIA INSTITUTE OF TECHNOLOGY UNIVERSITY OF CALIFORNIA MONTANA STATE UNIVERSITY

UNIVERSITY OF NEVADA, RENO NEW MEXICO STATE UNIVERSITY OREGON STATE UNIVERSITY

\author{
UNIVERSITY OF OREGON \\ UNIVERSITY OF SOUTHERN CALIFORNIA \\ STANFORD UNIVERSITY \\ UNIVERSITY OF AAWAII \\ UNIVERSITY OF TOKYO \\ UNIVERSITY OF UTAH \\ WASHINGTON STATE UNIVERSITY \\ UNIVERSITY OF WASHINGTON
}

The Supporting Institutions listed above contribute to the cost of publication of this Journal, but they are not owners or publishers and have no responsibility for its content or policies,

Mathematical parers intended for publication in the Pacific Journal of Mathematics should be in typed form or offset-reproduced, (not dittoed), double spaced with large margins. Please do not use built up fractions in the text of the manuscript. However, you may use them in the displayed equations. Underline Greek letters in red, German in green, and script in blue. The first paragraph or two must be capable of being used separately as a synopsis of the entire paper. Please propose a heading for the odd unmbered pages of less than 35 characters. Manuscripts, in triplicate, may be sent to any one of the editors. Please classify according to the scheme of Math. Reviews, Index to Vol. 39. Supply name and address of author to whom proofs should be sent. All other communications should be addressed to the managing editor, or Elaine Barth, University of California, Los Angeles, California, 90024 .

50 reprints to each author are provided free for each article, only if page charges have been substantially paid. Additional copies may be obtained at cost in multiples of 50 .

The Pacific Journal of Mathematics is issued monthly as of January 1966, Regular subscription rate: $\$ 114.00$ a year (6 Vol., 12 issues). Special rate: $\$ 57.00$ a year to individual members of supporting institution.

Subscriptions, orders for numbers issued in the last three calendar years, and changes of address shoud be sent to Pacific Journal of Mathematics, P.O. Box 969, Carmel Valley, CA 93924, U.S.A. Old back numbers obtainable from Kraus Periodicals Co., Route 100, Millwood, NY 10546.

\section{PUBLISHED BY PACIFIC JOURNAL OF MATHEMATICS, A NON-PROFIT CORPORATION}

Printed at Kokusai Bunken Insatsusha (International Academic Printing Co., Ltd.). 8-8, 3-chome, Takadanobaba, Shinjuku-ku, Tokyo 160, Japan.

Copyright (C) 1982 by Pacific Journal of Mathematics Manufactured and first issued in Japan 


\section{Pacific Journal of Mathematics}

Vol. 100, No. $2 \quad$ October, 1982

Kenneth F. Andersen, On the transformation of Fourier coefficients of

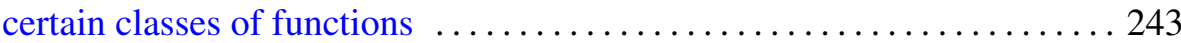

Steven Albert Bleiler, Realizing concordant polynomials with prime knots

Reinhard Bürger, Functions of translation type and solid Banach spaces of functions

Ulrich Daepp, The saturation of $k$-analytic rings and topological equivalence of associated analytic set germs .................. 271

Persi W. Diaconis and David Amiel Freedman, On the maximum difference between the empirical and expected histograms for sums . . . 287

David Amiel Freedman, On the maximum of scaled multinomial

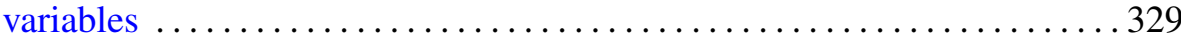

Persi W. Diaconis and David Amiel Freedman, On the difference between the empirical histogram and the normal curve, for sums. II ......... 359

Persi W. Diaconis and David Amiel Freedman, On the mode of an

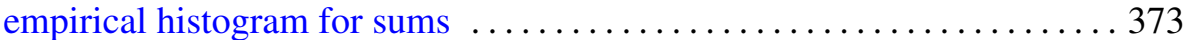

Jutta Hausen, Supplemented modules over Dedekind domains 387

Elyahu Katz, A moduli representation for the classification of twisted tensor

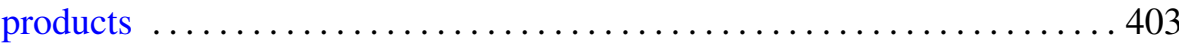

H. C. Madhekar and N. K. Thakare, Biorthogonal polynomials suggested by the Jacobi polynomials

Ted R. Pettis, Collections of covers of metric spaces 425

Ryōtarō Satō, Maximal functions for a semiflow in an infinite measure space

Michael Jay Stob, Invariance of properties under automorphisms of the lattice of recursively enumerable sets 